\title{
A Pilot Study of Myoelectrically Controlled FES of Upper Extremity
}

\author{
Rune Thorsen, Raffaella Spadone, and Maurizio Ferrarin, Member, IEEE
}

\begin{abstract}
Functional electrical stimulation (FES) of upper limbs can be used for the recovery of some hand functions on patients with CNS lesions. This study deals with the control of FES by means of myoelectrical activity detected from voluntarily activated paretic muscles. The specific aim of this paper is to evaluate the accuracy of myoelectrical control in terms of produced force and movement.

For this purpose, a specific device called myoelectrical controlled functional electrical stimulator (MeCFES) has been developed and applied to six tetraplegic patients with a spinal cord lesion and one stroke hemiplegic patient.

Residual myoelectric signals from the paretic wrist extensor (m. extensor carpi radialis, ECR) have been used to control stimulation of either the wrist extension (i.e., the same muscle) or thumb flexion. A tracking test based on a visual feedback of the produced force or movement compared to a reference target trajectory was used to quantify control accuracy. A comparison was made between the tracking performances of each subject with and without the MeCFES and the learning process for two of the subjects were observed during consecutive sessions.

Results showed that the wrist extension was improved in three out of five C5 SCI patients and the thumb flexion was largely increased in one incomplete $\mathrm{C3}$ SCI patient. The hemiplegic patient showed limited thumb control with the MeCFES but indicated the possibility of a carry over effect. It was found that a low residual natural force resulted in a less accurate movement but also with a large increase (up to ten times) of the muscle output. On the contrary, persons with a medium residual force obtained a smaller amplification of muscle force with a higher tracking accuracy.
\end{abstract}

Index Terms-EMG, functional electrical stimulation, grasp, hemiplegia, myoelectric control, SCI, tetraplegia.

\section{INTRODUCTION}

A COMPLETE C5/6 spinal cord injury (SCI), incomplete SCI, or cerebro-vascular stroke may lead to the loss of the functionality of one or both hands. Part of this population may have insufficient wrist extension and no grasp left. Restoration of the hand function will provide more independence and ability to perform activities of daily living (eating, drinking, writing, etc.), thereby significantly increasing the quality of life.

Extension of the wrist causes flexion of the fingers due to the passive forces in the finger flexors. This so-called tenodesis

Manuscript received March 10, 2000; revised February 23, 2001 and March 10,2001 . This work was supported by the European project TMR-NEUROS2 and by the Academy of Technical Sciences, Denmark. The device was built at Asah Medico A/S, Valseholmen 11-13, Hvidovre, Denmark, and tested at University Hospital, Rigshospitalet, Denmark.

R. Thorsen is with the Centro di Bioingegneria, Fond. Don Gnocchi-Politecnico di Milano, I-20148 Milano, Italy. He is also with the University of Twente, The Netherlands, and University College London, London, U.K.

R. Spadone and M. Ferrarin are with the Centro di Bioingegneria, Fond. Don Gnocchi-Politecnico di Milano, I-20148 Milano, Italy.

Publisher Item Identifier S 1534-4320(01)04457-6. grip [1] is a grasp that is often used and may be strengthened by enhancing the wrist extension force and by shortening the finger flexors [2]. Strengthening of the wrist extension can be done by surgical interventions and/or by functional electrical stimulation (FES) [3].

Several FES systems have recently been developed in order to improve the hand function in paralyzed patients. The Bionic Glove [4], [5] from the University of Alberta, Canada, is a hybrid system consisting of a garment with FES electrodes that uses a mechanical measurement of wrist extension to trigger stimulation of finger flexion. A commercially available product called the Handmaster [6] produced by NESS Ltd., Israel, is an orthosis in which stimulation electrodes are fitted; the orthosis locks the wrist in normal position. The Handmaster is controlled by a push-button on the control box to initiate a finger-extension/flexion stimulation sequence for either training or grasping. Both these systems use on/off stimulation with a preset value. A different approach is used by the Freehand system, made by NeuroControl Corp., Valley View, OH, USA [7]. This is an implanted system controlled by the contralateral shoulder which offers the possibility of muscle-selective stimulation and is "invisible," freeing the user from having to don and doff surface electrodes. Disadvantages include the complications of the invasive procedure, difficulties with repositioning of misplaced or defect electrodes, infections, etc. The controls of the Freehand system and the Handmaster are not related to the normal movements included in the motor function, whereas the Bionic Glove uses mechanical sensors to detect wrist extension and the control is therefore directly coordinated by the grasping sequence. The wrist extension strength, however, may be insufficient for this approach and the work described in this paper will show how the myoelectric signals from the wrist extensors may be used for the control of the stimulation.

Myoelectrical control has been used for on/off control or proportional control of prosthetics for some years [8], [9] and several research systems using myoelectrical control of FES have been reported [10]-[15]. It has been compared with other methods for control of the implant grasp system [16] and found feasible.

It has been demonstrated that the grasp can be augmented by stimulating the finger and thumb flexors using on/off control by the myoelectrical signals from wrist extension [10]. The principle of direct myoelectrical control of FES has been used to increase wrist extension from absent to a high force in some C5 SCI patients using an elaborate laboratory system [12].

Reliability and accuracy of the FES augmented movement is important for the grasp function. Reliability means that the object, such as a cup of hot coffee, does not slip out of the hand. 
Accuracy is necessary to avoid crushing fragile objects and to minimize the stimulation intensity, and thereby fatigue. An important issue is that the user can learn to use the limited information content [17] of the myoelectric signal, as a continually reliable and accurate control of the electrical stimulation [18], having only visual feedback and experience.

The transformation of the myoelectric signal into a reliable control signal is therefore also of great importance. The stimulation artefacts have to be reduced which can be done by a special amplifier configuration [19] and digital signal processing [12], [13], [20].

The myoelectric signal is a nonstationary stochastic signal [21], which makes it complicated to use as a reliable control signal. Several advanced methods such as classification [22], [23], autoregressive modeling [24], [25], ceptral-coefficients [26], or artificial neural networks [27]-[29] have been investigated but the traditional methods are the averaged rectified value (ARV) or root mean square (rms) over a moving window of the raw signal. ARV and rms are found to be increasing with increasing muscle contraction [30] but it is still somewhat unclear which one is the better [31], [32].

A continuous control of FES implies that the stimulation is a monotone function (e.g., linear) of the myoelectric signal. On/off control and level control are subclasses of continuous control where the control-stimulation relation is a piecewise linear function. Tests with the stimulation of wrist extensors using the myoelectrical control signal from the same muscle [20] showed that two tetraplegics could use only on/off control where a third tetraplegic could control four levels.

The aim of this study is to assess the feasibility of a continual myoelectrical control of FES to provide or improve wrist extension and/or thumb force for grasping. The final objective is to enable the user to control the movement as accurately as possible, therefore the focus of this paper is on the accuracy of the myoelectrically controlled functional electrical stimulated muscle contraction compared with the natural, i.e., unassisted, contraction. Accuracy tests has to be performed on patients with muscle paralysis since FES does not increase the muscle force in normal subjects. The functional aspects of this system have been assessed in another study [13].

\section{METHODS}

\section{A. Myoelectrically Controlled Stimulation System}

The myoelectrical controlled functional electrical stimulator (MeCFES) is a prototype device produced in ASAH Medico $\mathrm{A} / \mathrm{S}$, Denmark, for this research purpose. It comprises an amplifier, digital signal processor, and a stimulator. It can interface with a computer to allow monitoring and storage of the signals. The recorded signal is amplified and bandpass filtered to $10-500 \mathrm{~Hz}$ before sampling at $1 \mathrm{kHz}$. As the recorded signal is highly influenced by the stimulation pulses the amplifier is designed specially to suppress stimulation artefacts and their transients, as described in more detail by Thorsen 1999 [19]. Stimulation pulses are delivered with a fixed interval of $60 \mathrm{~ms}$ as amplitude modulated rectangular bi-phasic current pulses with a 0.3 -ms positive, 0.3 -ms zero and 0.3 -ms negative output. The stimulation responses, such as the stimulation artefacts and
M-waves, are dominating the amplified signal and cause a low signal-to-noise ratio (SNR), estimated to be less than $-20 \mathrm{~dB}$. To suppress the harmonics of the stimulation frequency and enhance the SNR a digital comb FIR-filter is applied. The filter equation is as follows:

$$
y_{n}=x_{n}-x_{n-d}
$$

where

$x \quad$ discrete recorded signal;

$n$ sample number;

$d$ number of samples in the stimulation interval (e.g., sampling frequency divided by stimulation frequency); $y \quad$ filter output.

After each stimulation pulse the signal is zeroed for the first 20 $\mathrm{ms}$ which leaves $40 \mathrm{~ms}$ of myoelectric signals in each stimulation interval. A subsequent bandpass filter emphasises frequencies of $100-300 \mathrm{~Hz}$ and the ARV over $1.2 \mathrm{~s}$ of the filtered signal is calculated and subtracted by an offset (background noise) [33]. This is used as the control signal which is multiplied by a gain factor to obtain the stimulation amplitude. The stimulation output is limited to between zero and an upper limit to protect the subject from excessive stimulation.

In our experiments the gain was found by trial and error until the subject could control the stimulation intensity continually and the upper stimulation limit was set at the maximal sustainable level but not above the force saturation level.

\section{B. Electrode Configurations and Sensors}

Surface electrodes, $\mathrm{Ag}-\mathrm{AgCl}$ electrodes from Medicotest A/S, Denmark, are used for detecting myoelectrical signals from the paretic but not completely paralyzed extensor carpi radialis muscle (ECR). An active reference electrode is used to increase the common mode noise suppression [34]. The MeCFES calculates and generates the stimulation current, delivered through surface electrodes (Pals, Axelgaard Manufacturing Co. Ltd., Fallbrook, CA, USA).

We have selected wrist extension to control stimulation of two useful motor functions: Wrist extensor Controlled Wrist extensor Stimulation (WCWS), and Wrist extensor Controlled Thumb flexor Stimulation (WCTS).

In WCWS, the stimulation electrodes are placed over the motor points of ECR. It is not necessary to distinguish between ECR longus and ECR brevis, but in terms of functionality, however, it means that in this case the controlling muscle and the stimulated muscle are the same. To minimize the stimulation artefacts the recording electrodes are placed on the muscle bulk perpendicular to the stimulation electrodes.

Evaluation of the wrist extension was performed with the subject's forearm supported by a little plate placed in a comfortable position for the subject. A lever, resting on the knuckles of the hand, was hinged to the plate with the axis coincident with the wrist. Two different measurements were taken: 1) Wrist extension against gravity measured by the unconstrained lever using a potentiometer to register the angle of the lever. The angle was measured from the resting position of the hand hanging loose. 2) Isometric force of wrist extension against the lever with the lever locked in an anatomically normal position. The force was 


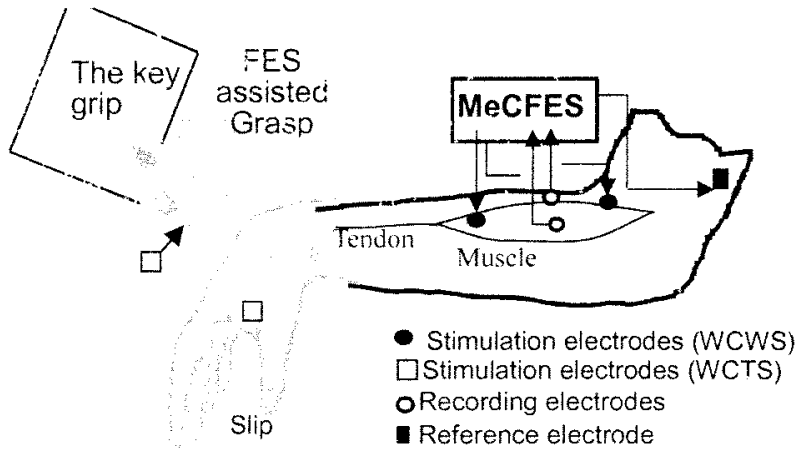

Fig. 1. The lateral pinch grip aided by the MeCFES with the electrode locations for the WCWS and WCTS configurations indicated.

measured with a strain-gauge transducer between the lever and the hand.

In the WCTS configuration the stimulation is applied at the thenar space to give thumb flexion while the recording electrodes are placed over the midline of the ECR muscle bulk; the stimulation and recording areas are separated, reducing the stimulation artefacts. A force transducer was placed between the subject's thumb and index finger, with the hand resting on a table in front of the subject. This test was performed under isometric condition.

The positioning of the electrodes for both configurations are indicated in Fig. 1.

\section{Experiment Design}

Preliminary experiments have shown that FES (and thereby MeCFES) of a normal muscle does not provide any additional force to maximal voluntary contraction. As a result, normal subjects cannot be used to evaluate the precision or functionality of MeCFES.

A convenience sample of 14 tetraplegics and one stroke patient, which were already involved in other FES trials, has been screened for the test. Six tetraplegics complied with the selection criteria (i.e., reduced wrist extension force with detectable residual EMG activity, good response to stimulation, and no medical contraindications). Table I lists the main data of the participants and the applied electrode configuration.

We have applied a tracking test as an objective measure of the controllability of the movement, first without and then with the MeCFES, so the subjects act as their own control. A target trajectory was shown on a computer screen and the subject was instructed to track it in real time with a marker. The marker would move horizontally across the screen with a constant speed and a vertical displacement proportional to isometric force or wrist angle. The test was intended to measure the controllability of a dynamic movement and the ability to maintain a constant level, therefore a trapezoidal target was chosen (see Fig. 3, dashed line).

We have defined the tracking error as the root mean square of the difference between the target and the track. The maximum level for the target was arbitrarily chosen at $90 \%$ of maximal MeCFES assisted output in the WCWS test and 75\% for the WCTS tests. The use of a 75\% target amplitude yields a higher theoretical tracking error in case of on/off control and a lower
TABLE I

Test Panel with Time Since Lesion

(TSL) AND CONFIGURATION USED to STIMULATE (SUbJeCt GG HaS FOREARM PARESIS FOLLOWING STROKE)

\begin{tabular}{|c|c|c|c|c|c|}
\hline ID & Lesion & $\overline{S e x}$ & $\begin{array}{l}\text { TSL } \\
\text { (yrs) }\end{array}$ & $\begin{array}{l}\text { Age } \\
\text { (yrs) }\end{array}$ & Config. \\
\hline $\mathrm{EG}$ & $\begin{array}{c}\text { C5- } \\
\text { Complete }\end{array}$ & $\mathrm{F}$ & 12 & 41 & WCWS \\
\hline$\overline{\mathrm{AA}}$ & \begin{tabular}{|c|}
$\mathrm{C} 5$ \\
Complete
\end{tabular} & $F$ & 2 & 79 & WCWS \\
\hline $\mathrm{FB}$ & \begin{tabular}{|c|}
$\mathrm{C} 5$ \\
Complete
\end{tabular} & $\mathrm{M}$ & 37 & 54 & WCWS \\
\hline$\overline{\mathrm{KGN}}$ & $\begin{array}{c}\text { C5- } \\
\text { Complete }\end{array}$ & $\mathrm{M}$ & 12 & 32 & WCWS \\
\hline $\mathrm{KN}$ & $\begin{array}{c}\mathrm{C} 5- \\
\text { Complete }\end{array}$ & $\bar{F}$ & 4 & 32 & WCWS \\
\hline $\overrightarrow{\mathrm{MF}}$ & $\begin{array}{c}\mathrm{C} 3 / 4- \\
\text { Incomplete }\end{array}$ & $\mathrm{M}$ & 8 & 25 & WCTS \\
\hline$\overline{\mathrm{GG}}$ & Stroke & $F$ & 1 & 30 & WCTS \\
\hline
\end{tabular}

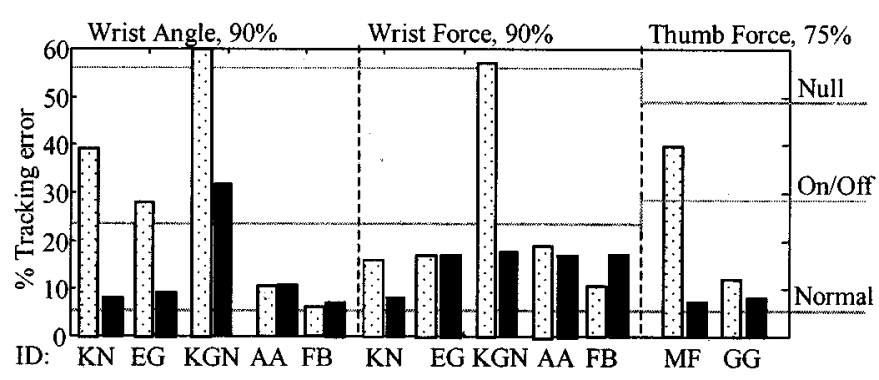

Fig. 2. Tracking error for the subjects. Under the subject ID is shown the maximum measured during volitional contraction with the MeCFES. The light bars show the unassisted errors and the dark bars show the MeCFES assisted tracking errors. The normal line shows the 5\% error that we expect of normal control. The on/off line represents the error an on/off control would have with the best possible timing and the null line represents the error of a zero tracking. For MF and GG the results of the best session are shown.

error in case of a null output. For both tests the total duration was $20 \mathrm{~s}$. During the development of the system and the WCWS protocol subject $\mathrm{KN}$ tested the device in five sessions where the last session is used for the reporting here. Subjects EG, KGN, $\mathrm{AA}$, and FB attended only one session each with the WCWS protocol, whereas MF and GG attended six and four sessions, respectively, with the WCTS protocol.

\section{RESULTS}

Restoration of normal movements is our ultimate goal and we will therefore use normal tracking as the reference. Tests on three male subjects without any neurological deficits have shown tracking errors below 5\% and without any deviation of more than $10 \%$ from the target, after less than three trials without applying the MeCFES. Wrist extension forces up to $100 \mathrm{~N}$ and thumb forces up to $50 \mathrm{~N}$ could be obtained.

A comparison between tracking errors with and without MeCFES are summarized in Fig. 2 for all the subjects, together with the errors of: normal tracking (5\%), theoretical tracking with on/off control, and theoretical tracking with null output. Note that the last two values change with the maximal value of the target. The figure shows the results of the tracking test recorded after tuning of the MeCFES system for WCWS configuration and the best results from the WCTS configuration. 


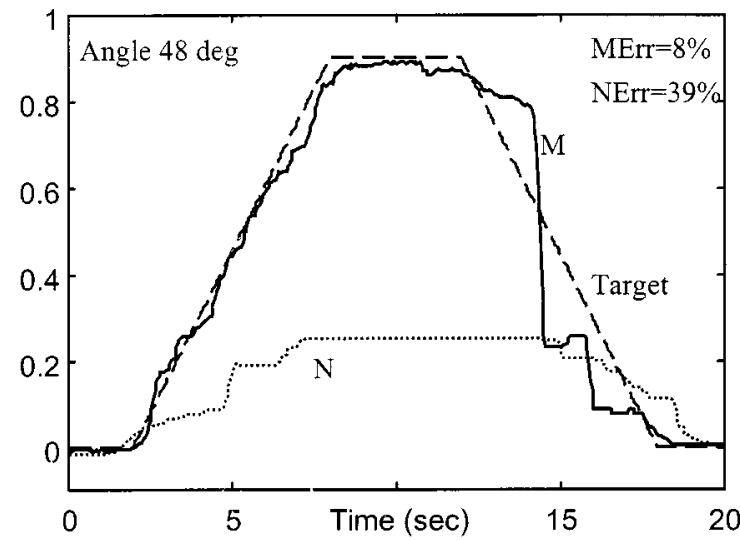

(a)

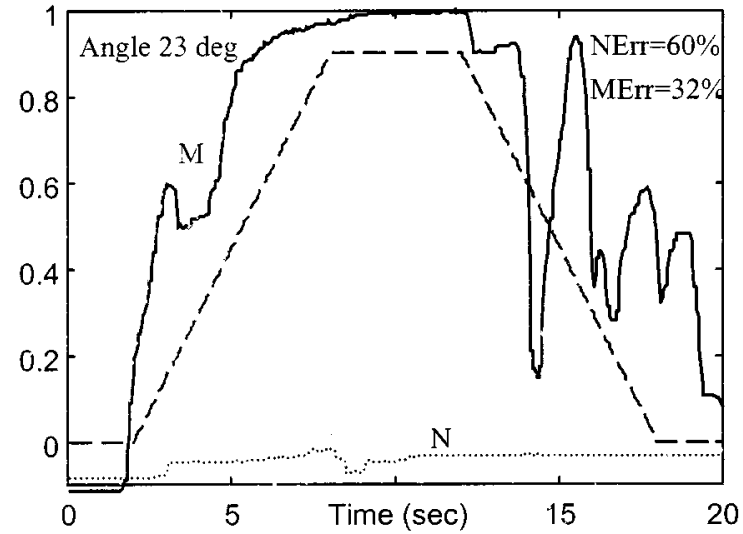

(b)

Fig. 3. Angle tracking using WCWS. The natural $(N$ : dotted) and the MeCFES assisted ( $M$ : solid) angle tracking for three tetraplegics are shown together with the target (dashed). (a) Subject KN. (b) Subject KGN.

In the following paragraphs, selected test results will be described for each considered configuration.

\section{A. WCWS Angle Tracking Tests}

In two (AA, FB) of the five subjects, the wrist extensor muscles were able to voluntary extend the wrist fully against gravity and therefore the MeCFES did not augment the angle range. In the other three subjects (KN, EG, KGN) who yielded weaker wrist extensors, the MeCFES gave an increased range of movement. Two tracking tests can be seen in Fig. 3. The maximal wrist angle was augmented by three and one-half times from $12^{\circ}$ to $43^{\circ}$ in subject $\mathrm{KN}$ [Fig. 3(a)], at a maximum current of $15 \mathrm{~mA}$. The subject had been trying the MeCFES in five sessions during the development of the system and had, therefore, gained some experience in using it. Notice how the ascending slope is followed closely whereas the descending slope, or the release part, reveals difficulties in controlling the movement.

Subject EG, who had a stronger extensor muscle than KN, demonstrated similar performances with the MeCFES but with a better natural tracking. In this case, the maximal wrist angle was nearly doubled by the MeCFES from 18 to $34^{\circ}$ with the maximal stimulation set to $25 \mathrm{~mA}$. This person had only tried the MeCFES system once before this test.

Subject KGN was the participant with the least wrist force and, as it appears from the Fig. 3(b), natural extension was prac-

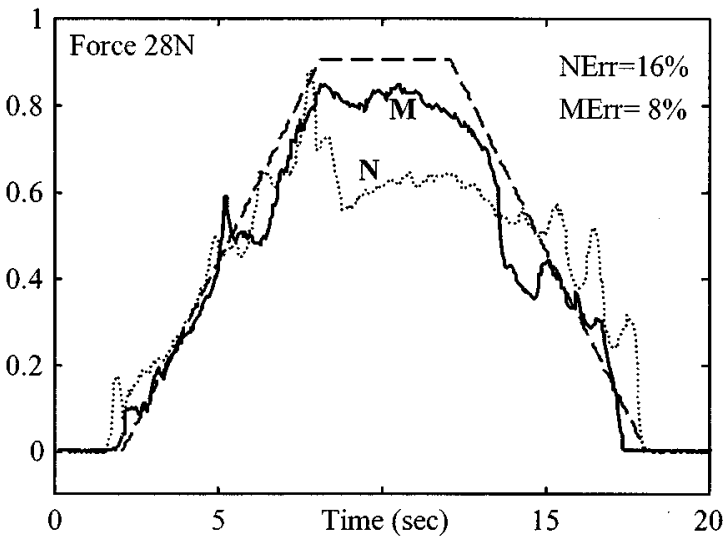

(a)

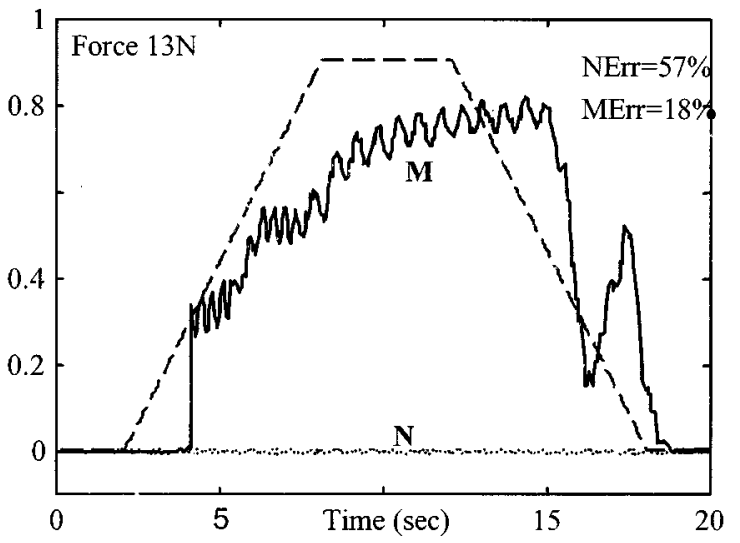

(b)

Fig. 4. Force tracking using WCWS. (a) KN. (b) KGN. The curves show the target (dashed), the MeCFES assisted tracking ( $M$ : solid) and the natural tracking $(N$ : dotted)

tically absent. With the MeCFES this person could produce a wrist angle of $23^{\circ}$; however, this was with the highest tracking error (Merr $=32 \%$ ) and therefore had the lesser control among the three subjects. It was the first and only time the person tried the device. Maximum stimulation amplitude was set to $20 \mathrm{~mA}$. Although the accuracy is poor, it proves that the MeCFES enables the subject to achieve an otherwise totally absent movement.

\section{B. WCWS Force Tracking Tests}

Wrist force enhancement was achieved in two (KN, KGN) of the five subjects using WCWS. These two are among the three of whom the MeCFES enhanced the wrist angle. Subject EG had an unassisted isometric force of $52 \mathrm{~N}$ with a tracking error of $17 \%$ and, in this case, the MeCFES did not give any change in performance.

The maximum stimulated force for subject KN was $28 \mathrm{~N}$ but, as can be seen in Fig. 4, the MeCFES enhances the short-term endurance, enabling the subject to maintain the force at the maximal level for a longer period of time than in an unassisted condition. As a consequence the error is halved by application of the MeCFES.

There was not a measurable natural wrist extension force of subject KGN, nevertheless the MeCFES provided $13 \mathrm{~N}$ isometric wrist extension force although with poor control as 


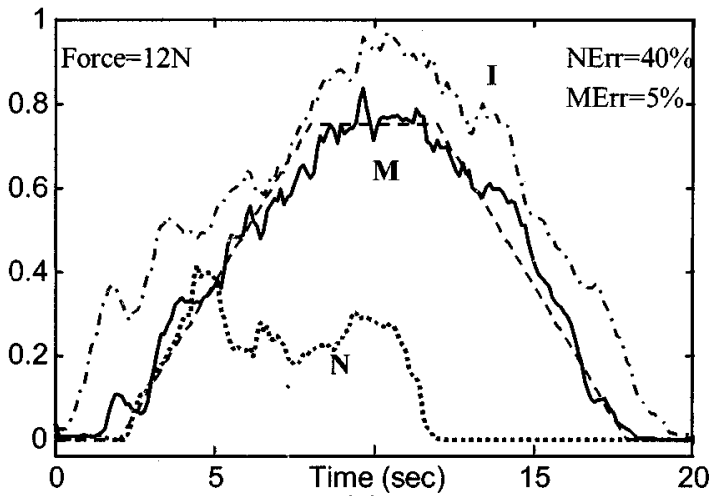

(a)

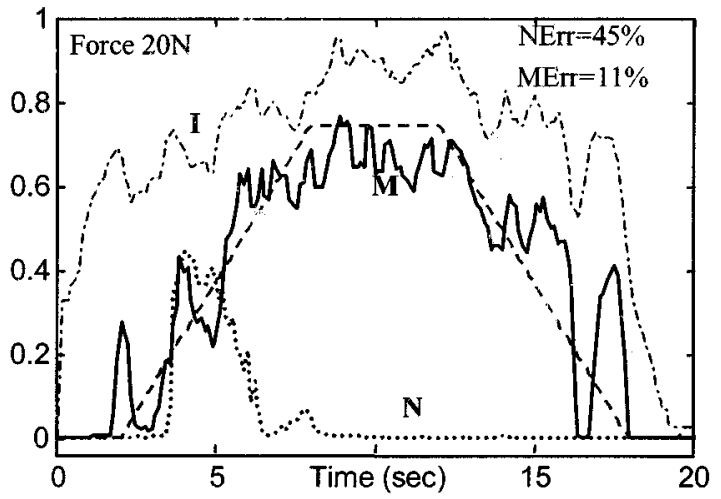

(b)

Fig. 5. Subject MF. $N$ : Natural force tracking. $M$ : WCTS. $I$ : Relative stimulation current. (a) The best tracking errors with WCTS. (b) Tracking errors with WCTS at a higher peak force.

shown in Fig. 4(b). Fatigue from the calibration trials may be the explanation why the force track does not reach the target amplitude, which was based upon the maximum amplitude at the start of the experiment.

\section{WCTS Tests}

An incomplete tetraplegic (MF) tried the WCTS configuration for six sessions on consecutive weekdays. We wanted to explore the effect of the optimization of MeCFES parameters and the possibility of learning. The best unassisted and best WCTS thumb force tracking from the first session are shown in Fig. 5. Notice that the unassisted contraction fatigues very rapidly. After one or two measurements of natural force it decreased to zero due to fatigue. When the MeCFES was applied the tracking required much less effort for the subject, although the MeCFES was applied after the unassisted tests. After the six sessions we noticed that the maximal augmented thumb force had increased from 12 to $20 \mathrm{~N}$ [Fig. 5(b)] and we increased the target amplitude accordingly. It should be noted that the tracking is much better in this case than for the WCWS tests. The best tracking error was $5 \%$, which is comparable with what we found in normal subjects.

In Fig. 5 we have plotted (with a dash-dot line) the relative stimulation current corresponding to the WCTS tracking. It can be seen that the stimulation is continually modulated but also that a stimulation level above $50 \%$ of maximum stimulation is needed to induce contraction. This can be attributed to the shape of the recruitment curve as reported in [35]. We have collected
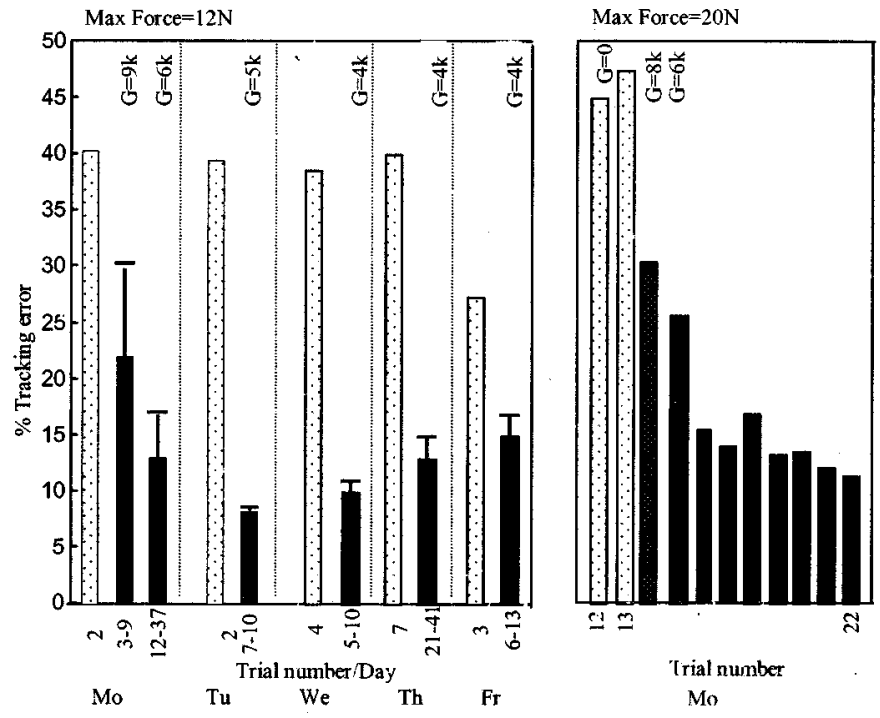

Fig. 6. WCTS tracking error for MF on consecutive weekdays. The gain $(G)$ has been changed in some tests and is shown above the bars. Grey bars: without MeCFES. Black bars: average of tracking errors with MeCFES with standard deviations on top. The test numbers are shown on the horizontal axis together with the name of the day. Tests used for adjusting the setup are omitted (i.e., missing numbers). In the table on the right, the results of individual tests from the last session are shown to underline the presence of possible learning effect.

the results from the six sessions with two days of rest before the last session in Fig. 6. The grey bars show the tracking error without MeCFES, which was measured first. The black bars show the average of the MeCFES aided thumb force tracking errors with standard deviation added on top. The trial numbers are shown on the horizontal axis together with the name of the day. Fig. 5(a) is from the Tuesday session and Fig. 5(b) is from the second Monday session. Some trials have been discarded because they were used to tune the system gain, other measurements were taken (e.g., recruitment curves) or because of erroneous trials (e.g., failing concentration). Data from the second Monday, shown on the right side of Fig. 6, are left raw to show that there is a local improvement which can be attributed to a learning effect. In general, in all the experiments, the major improvements of the tracking errors have been made during the first 2-3 tests, but at the same time the gain was being tuned. A further small reduction on the tracking error, however, was also observed for the subsequent trials, which can be attributed to learning. From the Wednesday to the first Monday the average error increased but the maximal force had almost doubled by the second Monday.

\section{Stroke Patient}

The hemiplegic person (GG) was not able to produce any wrist extension before the experiments although some myoelectric activity was present. Four WCTS sessions took place within a fortnight. At the initial session the maximal natural force of the thumb reached $14 \mathrm{~N}$ but with limited control, as can be seen in Fig. 7. In this first session, the best MeCFES augmented force tracking-error was $42 \%$, which was worse than the unassisted error of $16 \%$. It is apparent that the MeCFES gave rise to instability. Both the natural and the MeCFES assisted performances had improved by the fourth session, with an unassisted error of 


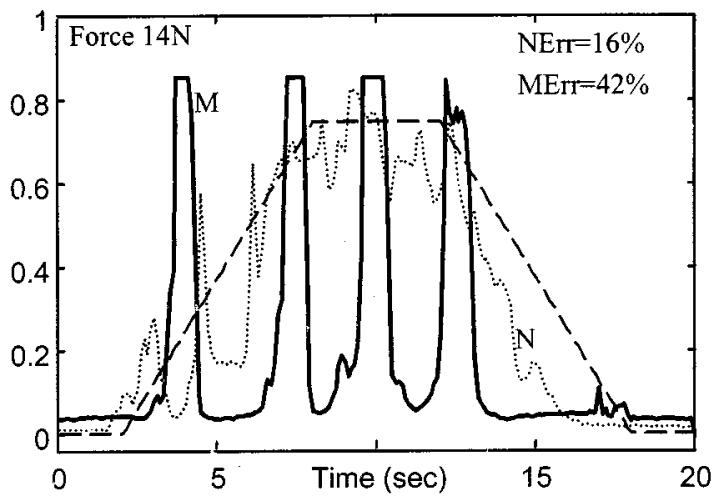

(a)

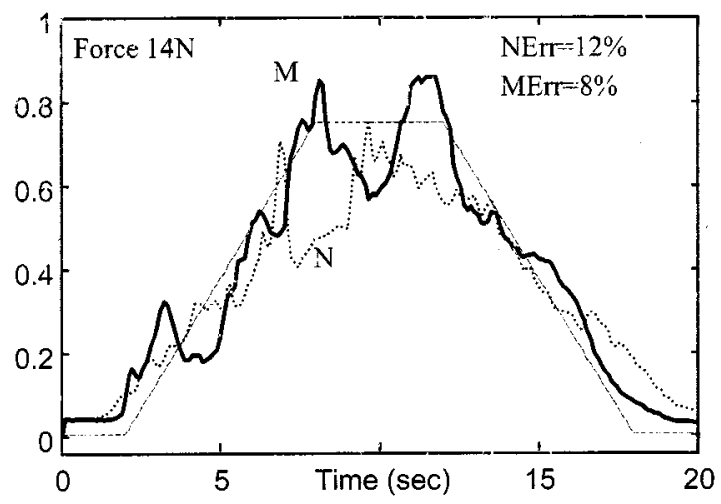

(b)

Fig. 7. Hemiplegic subject GG. (a) First session of thumb force tracking. (b) Fourth session. The curves are: the target (dashed), the MeCFES assisted tracking $(M$ :solid) and the natural tracking $(N:$ dotted $)$.

$12 \%$ and a better MeCFES tracking error of only $8 \%$ [Fig. 7(b)]. For the four sessions, the tracking errors are shown as a bar graph in Fig. 8. The MeCFES does not seem to have any noticeable influence on the tracking once the subject has become confident with using the MeCFES.

\section{DISCUSSION}

Subjects KGN and KN, who had weak wrist extension, gained an improvement in the tracking performances with MeCFES, in both wrist angle and force tracking tests compared with unassisted trials. There was not a noticeable increase of force for one subject (EG) but a better ability to control the wrist angle against gravity, resulting in a lower tracking error with the MeCFES. This subject had a tendon transfer, which might be the explanation for this.

Subjects AA and FB, who had the strongest natural contraction of wrist extensors and good angle tracking performances in unassisted conditions, produced no additional force when FES was applied within the acceptable stimulation intensity; therefore there was no improvement in the tracking error with the MeCFES.

It can be concluded that patients with the highest natural tracking error, due to low natural force, are good candidates for WCWS MeCFES. This was also the case in one SCI subject (MF) using WCTS.

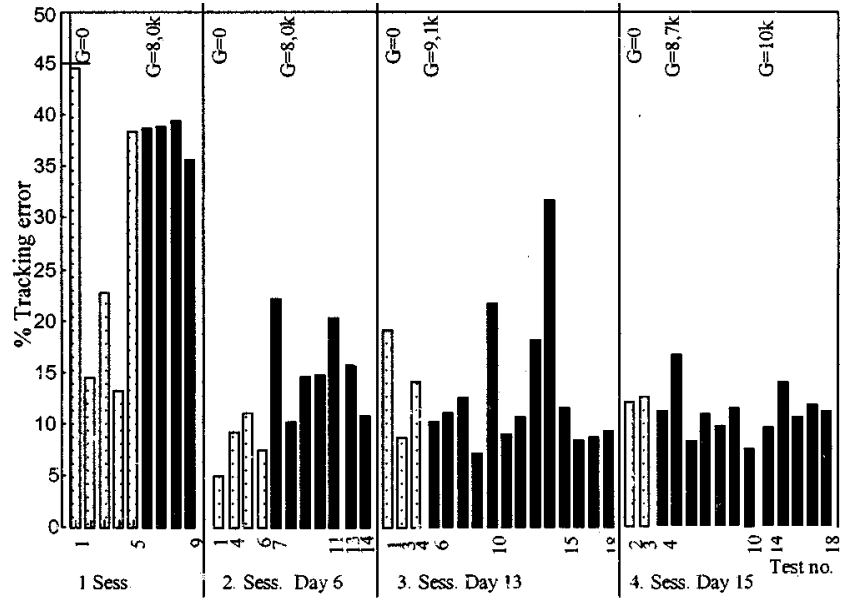

Fig. 8. Tracking error for hemiplegic subject (GG) on 4 different days. Grey bars are without and black bars are with WCTS. Date and test number are below the bars and the gain factors are above.

For both WCWS and WCTS configurations, the obtained tracking error with a continuous myoelectric control was better than the theoretic error with on/off control in all but one patient (KGN), who had the weakest wrist extension. For this subject the MeCFES assisted angle tracking error was worse than on/off control.

These results demonstrate the feasibility of a continuous control of FES using EMG signals in subjects with sufficient residual muscle contraction, whereas patients with very weak muscle contraction may only exhibit on/off control.

The stroke patient (GG) did not show any improvement with the WCTS compared to natural movement. A carry-over effect, however, is seen as an improvement of the WCTS performance, as well as without the device after the first session.

Using the MeCFES improved tracking accuracy, although the level of a normal subject was not reached. One reason for this is the lower number of volitionally controlled motor units of a paretic muscle compared to a normal muscle, which reduces the dynamic range of the controlling myoelectric signal. A number of phenomena involved in MeCFES assisted contractions can explain the reduction of accuracy. Control is better during an increasing muscle contraction than during the release phase. This may be attributed to hysteresis and other nonlinearities of the recruitment curve [35]. Normal movements are coordinated by agonist and antagonist muscles. During these experiments the antagonist muscles were neither stimulated nor under normal volitional control.

It is known that the stimulation can cause secondary responses, like the H-reflex and the F-response [36], which add to the recorded myoelectric signal. They reduce the signal to noise ratio and act as a positive feedback, thus causing instability.

Future system development should therefore confront the above items and consider signal processing methods, to suppress the secondary responses of stimulation, and adequate stimulation of the antagonist muscles (in WCWS configuration) to improve the accuracy of wrist movement control, in particular during the release phase. Moreover, it should be investigated to what extent learning can improve control and 
how long this process will take. This is even more important for stroke patients, as it has been shown that in these patients myoelectric biofeedback can be used to reeducate muscles [37], [38] and to augment the natural muscle force [39]. The MeCFES approach, presented here, is combining the expected benefits of both myoelectrical biofeedback and FES.

When translating the MeCFES into practical use it should be considered what effect forearm posture changes may have on the myoelectric signal and the effect of the FES in terms of fluctuations in signal level and induced force. In this basic study of the MeCFES, this has not been assessed since the arm was held in a fixed position. It should be considered in a clinical study of the MeCFES together with the influences of motion artifacts and electromagnetic fields. In case it induces a problem these matters may be met by incorporating the electrodes in a splint like the Handmaster.

We have initiated a study to test if the principle can be applicable to paretic muscles in the lower limb, for example, as a supplement to the dropped foot stimulator [40] and to further investigate the limiting factors for the reliability and accuracy of the enforced movement.

\section{CONCLUSION}

An MeCFES has been tested by six tetraplegics and one hemiplegic using a tracking test. The conclusion is that the myo-electrical signal can be used to control FES and thereby augment the motor output of paretic muscles affected by spinal cord injury. The prerequisite is that the muscle has a composition of innervated motor units where some are under natural control and some are not, as can be the case after spinal cord lesion. Three C5 tetraplegics had a weak wrist extension that could be augmented by the MeCFES. One incomplete SCI could use the MeCFES to let wrist extension control stimulation of the lateral pinch grip with a doubling of force and increased endurance. The tracking tests have shown that accuracy of the movement can be from on/off control to a very good control following a trapezoidal target.

The tracking tests have showed that an MeCFE-assisted movement can be continually controlled, but not with the same accuracy as a normal natural movement. The proposed concept is considered feasible because the controlling movement is a natural part of the tenodesis grip.

\section{ACKNOWLEDGMENT}

The authors with to thank the subjects who, with patience, have taken part in the experiments and Dr. Biering-Soerensen for the clinical support.

\section{REFERENCES}

[1] E. Moberg, The Upper Limb in Tetraplegia. Stuttgart, Germany: Georg Thieme, 1978.

[2] L. Harvey, "Principles of conservative management for a nonorthotic tenodesis grip in tetraplegics," J. Hand Therapy, vol. 9, pp. 238-242, 1996.

[3] M. W. Keith, K. L. Kilgore, P. H. Peckham, K. S. Wuolle, G. Creasey, and M. Lemay, "Tendon transfers and functional electrical stimulation for restoration of hand function in spinal cord injury," J. Hand Surg. Amer., vol. 21, pp. 89-99, 1996.
[4] M.G. Prochazka, M. Wieler, and Z. Kenwell, "The bionic glove: An electrical stimulator garment that provides controlled grasp and hand opening in quadriplegia," Arch. Phys. Med. Rehab., vol. 78, pp. 608-614, 1997.

[5] D. Popovic, A. Stojanovic, A. Pjanovic, S. Radosavljevic, M. Popovic, S. Jovic, and D. Vulovic, "Clinical evaluation of the bionic glove," Arch. Phys. Med. Rehabil., vol. 80, pp. 299-304, 1999.

[6] M. J. Ijzerman, T. S. Stoffers, and F. Groen et al., "The NESS Handmaster orthosis: Restoration of hand function in C5 and stroke patients by means of electrical stimulation," J. Rehab. Sci., vol. 9, pp. 86-89, 1996.

[7] K. L. Kilgore, P. H. Peckham, M. W. Keith, G. B. Thrope, K. S. Wuolle, A. M. Bryden, and R. L. Hart, "An implanted upper-extremity neuroprosthesis. Follow-up of five patients," J. Bone Joint Surg. Amer., vol. 79, pp. 533-541, 1997.

[8] P. A. Parker and R. N. Scott, "Myoelectric control of prostheses," Crit. Rev. Biomed. Eng., vol. 13, pp. 283-310, 1986.

[9] T. R. Scott, P. H. Peckham, and K. L. Kilgore, "Tri-state myoelectric control of bilateral upper extremity neuroprostheses for tetraplegic individuals,” IEEE Trans. Rehab. Eng., vol. 4, pp. 251-263, Dec. 1996.

[10] S. Saxena, S. Nikolic, and D. Popovic, "An EMG-controlled grasping system for tetraplegics," J. Rehab. Res. Dev., vol. 32, pp. 17-24, 1995.

[11] M. Rakos, B. Freudenschuss, W. Girsch, C. Hofer, J. Kaus, T. Meiners, T. Paternostro, and W. Mayr, "Electromyogram-controlled functional electrical stimulation for treatment of the paralyzed upper extremity," Artif. Organs, vol. 23, pp. 466-469, 1999.

[12] E.-U. Haxthausen, F. Biering-Sorensen, and K. Dahl et al., "Restoration of key grip in SCI patients, an attempt using FES controlled by emg from the stimulated muscle," presented at the New Theoretical and Applied Approaches in the Restoration of Impaired Motor Control, Milano, Italy, 1991.

[13] R. Thorsen, M. Ferrarin, R. Spadone, and C. Frigo, "Functional control of the hand in tetraplegics based on synergistic EMG activity," Artif. Organs, vol. 23, no. 5, pp. 470-473, 1999.

[14] D. C. Howson and J. E. Heule, "Electronic Circuit Permitting Simultaneous Use of Stimulating and Monitoring Equipment," U.S. Patent 4235242 , Nov. 25, 1980

[15] J. A. Hodgson, "Electrode Array for Muscle Stimulation and Recording," U.S. Patent 4619 266, 1986.

[16] R. L. Hart, K. L. Kilgore, and P. H. Peckham, "A comparison between control methods for implanted FES hand-grasp systems," IEEE Trans. Rehab. Eng., vol. 6, pp. 208-218, June 1998.

[17] L. Vodovnik and S. Rebersek, "Information content of myo-control sig nals for orthotic and prosthetic systems," Arch. Phys. Med. Rehab., vol. 55, pp. 52-56, 1974.

[18] L. Vodovnik, C. Long, J. Reswick, A. Lippay, and D. Starbuck, "Myoelectric control of paralyzed muscles," IEEE Trans. Biomed. Eng., vol. 12, pp. $169-172,1965$

[19] R. Thorsen, "An artefact suppressing fast-recovery myoelectric amplifier," IEEE Trans. Biomed. Eng., vol. 46, pp. 764-766, June 1999.

[20] S. Sennels, F. Biering-Sorensen, O. T. Andersen, and S. D. Hansen, "Functional neuromuscular stimulation controlled by surface electromyographic signals produced by volitional activation of the same muscle: Adaptive removal of the muscle response from the recorded EMG-signal," IEEE Trans. Rehab. Eng., vol. 5, pp. 195-206, June 1997.

[21] E. Kwatny, D. H. Thomas, and H. G. Kwatny, "An application of signal processing techniques to the study of myoelectric signals," IEEE Trans. Biomed. Eng., vol. BME-17, pp. 303-313, 1970.

[22] S. P. Lee, J. S. Kim, and S. H. Park, "An enhanced feature extraction algorithm for EMG pattern classification," IEEE Trans. Rehab. Eng., vol. 4, pp. 439-443, Dec. 1996.

[23] P. Herberts, C. Almstrom, R. Kadefors, and P. D. Lawrence, "Hand prosthesis control via myoelectric patterns," Acta Orthop. Scand., vol. 44, pp. 389-409, 1988.

[24] G. Hefftner, W. Zucchini, and G. G. Jaros, "The electromyogram (EMG) as a control signal for functional neuromuscular stimulation. Part I-II," IEEE Trans. Biomed. Eng., vol. 35, pp. 230-242, Apr. 1988.

[25] R. Knox, D. H. Brooks, and M. E. Manolakis, "New features based on alternative representations of AR models for upper limb EMG recognition," in Proc. Annu. Conf. Engineering Medicine Biology, vol. 15, 1993, p. 1138.

[26] W. J. Kang, J. R. Shiu, C. K. Cheng, J. S. Lai, H. W. Tsao, and T. S. Kuo, "Cepstral coefficients as the new features for electromyography (EMG) pattern recognition," in Proc. Annu. Conf. Engineering Medicine Biology, vol. 15, 1993, p. 1144. 
[27] A. Kostov, R. B. Stein, and W. W. Armstrong, "Learning of EMG-patterns by adaptive logic network," in Proc. Annu. Conf. Engineering Medicine Biology, vol. 15, 1993, p. 1136.

[28] J. D. Costa and R. E. Gander, "Classification of muscle contraction levels with an artificial neural network," in Proc. 15th Annu. Int. IEEE Engineering in Medicine and Biology Society Injury Conf., vol. 15, 1993, pp. 259-260.

[29] K. Kuribayashi, "A discrimination system using neural network for EMG controlled prosthesis," in Proc. IEEE/RSJ Int. Conf. Int. Robots Systems, 1993, pp. 1750-1755.

[30] C. J. DeLuca, "The use of surface electromyography in biomechanics," J. Appl. Biomech., vol. 13, pp. 135-163, 1997.

[31] E. A. Clancy and N. Hogan, "Probability density of the surface electromyogram and its relation to amplitude detectors," IEEE Trans. Biomed. Eng., vol. 46, pp. 730-739, June 1999.

[32] R. Thorsen, M. Ferrarin, J. Schuurmans, P. Veltink, and C. Frigo, "The myoelectric signal as control for functional electrical stimulation," in Proc. SENIAM - 4th Topical Workshop Hertogoenbosh, The Netherlands, 1999, pp. 140-145. Ser. Future App. Surface EMG.

[33] R. Thorsen, "Restoration of hand function in tetraplegics using myoelectrically controlled functional electrical stimulation of the controlling muscle," Ph.D. dissertation, Electronics Institute, The Technical University of Denmark, Lyngby, 1997.

[34] J. G. Webster, "Reducing motion artifacts and interference in biopotential recording," IEEE Trans. Biomed. Eng., vol. BME-31, pp. 823-826, 1984.

[35] W. K. Durfee and K. E. MacLean, "Methods for estimating isometric recruitment curves of electrically stimulated muscle," IEEE Trans. Biomed. Eng., vol. 36, pp. 654-667, July 1989.

[36] M. A. Fisher, "AAEM minimonograph \#13: H reflexes and F waves: Physiology and clinical indications," Muscle Nerve, vol. 15, pp. 1223-1233, 1992.

[37] R. E. Schleenbaker and A. G. Mainous, "Electromyographic biofeedback for neuromuscular reeducation in the hemiplegic stroke patient: A meta-analysis," Arch. Phys. Med. Rehab., vol. 74, pp. 1301-1304, 1993.

[38] G. H. Kraft, S. S. Fitts, and M. C. Hammond, "Techniques to improve function of the arm and hand in chronic hemiplegia," Arch. Phys. Med. Rehab., vol. 73, pp. 220-227, 1992.

[39] M. Dimitrijevic, S. Dobrivoje, S. Stokic, A. Wawro, and C. Wun, "Modification of motor control of wrist extension by mesh-glove electrical affetent stimulation in stroke patients," Arch. Phys. Med. Rehab., vol. 77 , pp. 252-258, 1996.

[40] P. Taylor, A. Dunkerley, D. Wood, and I. Swain, "Long term follow up of 160 users of the odstock dropped foot stimulator," in Vienna Int. Workshop Functional Electrostimulation, vol. 6, 1998, pp. 189-192.

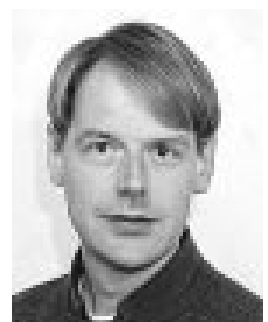

Rune Thorsen was born in Denmark, Lyngby, in 1967. He received the M.Sc.e.e degree from the Technical University of Denmark, Lyngby, in 1994 and the $\mathrm{Ph} . \mathrm{D}$. degree in bioengineering in 1997.

As a Researcher in the Danish company Asah Medico A/S, he carried out research for the European project EPCES (1994-1997), after which he started at three-year Post-doctoral position in the European project NEUROS (under the TMR program) where he worked in several countries: 1997 in Italy at Centro di Bioingegneria, Milano, 1998 in The Netherlands working at Universiteit Twente, and in 1999 conducting experimental work in collaboration with Salisbury District Hospital and University College London in the U.K. Since October 2000, he has been with Centro di Bioingegneria, Milano, Italy. His resarch interests include signal processing, electronics, functional electrical stimulation, and use of EMG signals for control.

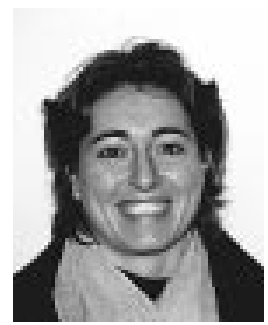

Raffaella Spadone was born in Milan, Italy, in 1974. She received the Dipl. degree in bioengineering from the Polytechnic of Milan in 1996.

She is a Researcher at the Bioengineering Center (Fond. Don Carlo Gnocchi IRCCS ONLUNS and Polytechnic of Milan) where she is a collaborator of the Laboratory for the Study of Motor Recovery (LaRMo). Her main research interests include functional electrical stimulation and innovative orthosis for motor recovery in paralyzed persons, clinical gait analysis, and spasticity evaluation.

Ms. Spadone is a member of the Italian Society of Clinical Movement Analysis (SIAMoC).

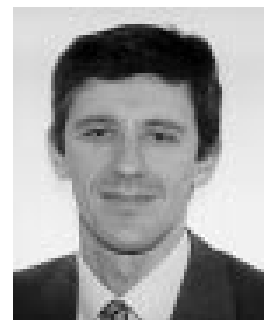

Maurizio Ferrarin (M'97) was born in Milan, Italy, in 1964. He received the M.Sc. degree in electronic engineering and the Ph.D. degree in bioengineering from the Polytechnic of Milan in 1989 and 1993, respectively.

$\mathrm{He}$ is a Researcher at the Bioengineering Centre (Fond. Don Carlo Gnocchi IRCCS ONLUS and Polytechnic of Milan) where he is responsible for the Laboratory for the Study of Motor Recovery (LaRMo). $\mathrm{He}$ is also a Professor of Rehabilitation Robotics at the Polytechnic of Milan. His main research interests include functional electrical stimulation and innovative orthosis for motor recovery in paralyzed persons, clinical gait analysis, biomechanics, spasticity evaluation, and ergonomics of wheelchair propulsion and of seat cushions.

Dr. Ferrarin is a member of the IEEE Engineering in Medicine and Biology Society (IEEE/EMBS), the International Functional Electrical Stimulation Society (IFESS), and the Italian Society of Clinical Movement Analysis (SIAMoC). 